\title{
A New modified particle swarm Optimization (PSO) Technique for Non-Convex Economic Dispatch
}

\author{
Ruchi Solanki ${ }^{1}$, N. P. Patidar ${ }^{2},{ }^{3}$ K. T. Chaturvedi \\ ${ }^{1}$ (Department of Electrical \& Electronics Engg, VNS Faculty of Engg. India) \\ ${ }^{2}$ (Department of Electrical Engg,NIT, Bhopal, India) \\ ${ }_{3}^{3}$ (Department of Electrical \& Electronics Engg,UIT-RGPV, Bhopal, India)
}

\begin{abstract}
This paper gives a new modified technique for evaluating non-convex economic dispatch (NCED) problem having non-linear, discontinuous cost function. This new particle swarm optimization technique incorporates time varying acceleration coefficients (NPSOTVAC). The highly non-linear cost function due to ramp rate limit, prohibited operating zones and valve point loading effect is considered in the proposed method. The effectiveness of proposed NPSOTVAC algorithm is proved through test on 6 and 15 unit test systems.
\end{abstract}

Keywords: Nonconvex economic dispatch (NCED), Particle swarm optimization (PSO), Time varying acceleration coefficients (TVAC), New PSO with Time-Varying Acceleration Coefficients (NPSOTVAC).

\section{Introduction}

The economic load dispatch (ELD) is about minimizing the total generation cost of generating units in order to meet the power demand while satisfying equality and inequality constraints. Traditionally, the cost function of the generator is assumed to be piecewise linear and represented by a quadratic function. However, in practical, this assumption is no longer valid due to the valve point effect and prohibited operating zones (POZ) of generating units. This makes the ELD problem highly non linear and nonconvex optimization problem, which is difficult to be solved by mathematical approach. Moreover, the ELD problem becomes more complicated when ramp-rate limits and transmission losses are taken into account.

Many optimization methods have been used for solving classical ELD problem, such as lambda iteration, gradient method, linear programming and quadratic programming [1]. Most of these methods might be unable to solve nonconvex and discontinuous ELD problem efficiently. This is because these methods require monotonically increasing incremental cost function, where the derivative information of the cost function exists. In order to solve nonconvex ELD problem, heuristic methods such as evolutionary programming (EP), genetic algorithm, artificial immune system, tabu search, ant colony optimization and particle swarm optimization (PSO) have been implemented to solve nonconvex and discontinuous ELD problems [2,3]. These methods do not require the derivative information of the cost function. Thus, it can be used to solve nonconvex ELD problem due to valve point effects, prohibited operating zones, multi fuels options and nonlinear power flow constraints that cannot be solve by classical methods. However, these methods not always guarantee the global optimal solution.

Among these methods, the particle swarm optimization (PSO) method is widely used for solving ELD problem due to its simple implementation, less memory storage and able to find global solution. Many modifications and hybrid of PSO methods were proposed for solving the nonconvex ELD problem such as IPSO [8], fuzzy adaptive PSO [12], simulated annealing PSO (SA- PSO) [14], SOH-PSO [15],adaptive PSO (APSO) [16] and an improved coordinated aggregation based PSO (ICA-PSO) [17]. Nevertheless, the classical PSO can be further improved to obtain a good solution.

The PSO, first introduced by Kennedy and Eberhart [9] is a flexible, robust, population based stochastic search/optimization algorithm with inherent parallelism. In recent years this method has gained popularity over its competitors and is increasingly gaining acceptance for solving economic dispatch [6-8] and a variety of power system problems [13], due to its simplicity, superior convergence characteristics and high solution quality. The convergence towards the global best solution is governed by the proper control of global and local exploration capability. The concept of inertia weight was introduced to balance the local and global search. A high inertia weight during initial part of the search ensured global exploration while a lower value at the end facilitates global convergence. With this in view, the concept of time varying inertia weight (TVIW) was introduced in $[10,11]$.

A novel PSO based strategy based on time varying acceleration is applied in this paper for the NCED to overcome the problem of premature convergence and for finding the global optima. A relatively high value of the cognitive component results in excessive wandering of particles while a higher value of the social component causes premature convergence of particles [6]. Hence, time-varying acceleration coefficients 
(TVAC) [18] are employed to strike a proper balance between the cognitive and social component during the search. This paper proposes a new modified PSO based algorithm, Integration of this approach with time varying penalty helps in exploring the search space very effectively to identity the promising solution region. The NPSOTVAC method is illustrated through two systems with 6 and 15 generating units.

\section{II.Objective Function Formulation}

II.1Economic cost function: The primary objective of any ED problem is to reduce the operational cost of system fulfilling the load demand within limit of constraints. Simplified economic dispatch problem can be represented as a quadratic fuel cost objective function as described in Eq. (1)

$$
\begin{gathered}
\operatorname{MinF}_{T}=\sum_{i=1}^{N} \boldsymbol{F}_{i}\left(\boldsymbol{P}_{\boldsymbol{i}}\right) \\
F_{i}\left(P_{i}\right)=\sum_{i=1}^{N}\left(a_{i} P^{2}+b_{i} P+c_{i}\right) R s / h
\end{gathered}
$$

Where $F_{T}$ : total generating cost; $F_{i}$ : cost function of $i$ th generating unit; $a_{i}, b_{i}, c_{i}$ : cost coefficients of generator $i$; $P_{i}$ : power of generator $i$; $\mathrm{N}$ : number of generators.

\section{II.2.Economic cost function with valve point loading effect.}

The generating units with multiple valves in steam turbines are available. The opening and closing of these valves are helpful to maintain the active power balance. However it adds the ripples in the cost function as shown in Fig. 1 which makes the objective function highly nonlinear. The sinusoidal functions are added to the quadratic cost function as given in (2)

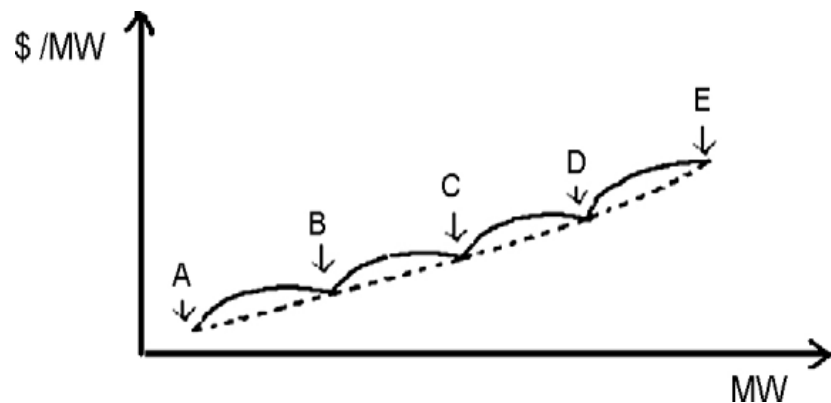

Figure 1. Incremental fuel cost curve for 5 valve steam turbine unit

$$
F_{i}\left(P_{i}\right)=a_{i} P_{i}^{2}+b_{i} P_{i}+c_{i}+\left|k_{i} \times \sin \left(l_{i} \times\left(P_{i}^{\min }-P_{i}\right)\right)\right|
$$

Where $k_{i}$ and $l_{i}$ are the coefficients of generator $i$ considering valve point loading effect.

\section{Equality constraint and Inequality constraints:}

(1) System power balance equation: It is an equality constraint which should be satisfied for power plant system.

$$
\sum_{i=1}^{N} P_{i}-\left(L_{D}+P_{L}\right)=0
$$

Where $L_{D}$ and $P_{L}$ are load demand and power losses respectively. To calculate system losses, methods based on penalty factors and constant loss formula coefficients or B-coefficients [1] are in use. The latter is adopted in this paper as per which transmission losses are expressed as

$$
P_{L}=\sum_{i=1}^{N} \sum_{j=1}^{N} P_{i} B_{i j} P_{j}+\sum_{i=1}^{N} B_{o i} P_{i}+B_{o o}
$$

(2)Power generation capacity limits:

$$
P_{i}^{\min } \leq P_{i} \leq P_{i}^{\max } \quad i=1,2, \ldots, N
$$

where $P_{i}^{\min }$ and $P_{i}^{\max }$ are minimum and maximum power generation capacity limit of $i^{\text {th }}$ generator 


\section{Prohibited operating zone:}

The generators may have certain range where operation is restricted due to the physical limitation of machine component, steam valve, vibration in shaft bearing etc as shown in Fig. 2. The consideration of prohibited operating zone creates discontinuities in cost curve and converts the constraint as below.

$$
\boldsymbol{P}_{i} \in\left\{\begin{array}{l}
\boldsymbol{P}_{i}^{\min } \leq \boldsymbol{P}_{i} \leq \boldsymbol{P}_{i 1}^{L} \\
\boldsymbol{P}_{i k-1}^{U} \leq \boldsymbol{P}_{i} \leq \boldsymbol{P}_{i k}^{L} \\
\boldsymbol{P}_{i k}^{U} \leq \boldsymbol{P}_{i} \leq \boldsymbol{P}_{i}^{\max }
\end{array}\right.
$$

Here $k$ are the number of prohibited zones in $i$ th generator curve, $\boldsymbol{P}_{i k}^{L}$ is the lower limit of $k$ th prohibited zone, and $\boldsymbol{P}_{i k}^{U}$ is the upper limit of $k$ th prohibited zone of $i$ th generator.

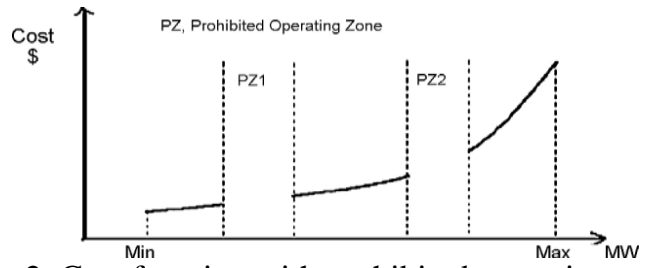

Figure 2. Cost function with prohibited operating zone.

\section{Generator ramp-rate limits}

The operating range of on-line generating units is restricted by ramp-rate limits. In practice, the unit output can not be adjusted instantaneously whenever load changes. Ramp rate limits, i.e. up-rate limit $U R_{i}$, down-rate limit $D R_{i}$ and previous hour generation $P_{i}^{o}$ restrict the operating region of all the on-line units. When the generator ramp-rate limits are considered, the operating limits of the $i^{\text {th }}$ generating unit are modified as follows:

$$
\operatorname{Max}\left(P_{i}^{\min }, P_{i}^{o}-D R_{i}\right) \leq P_{i} \leq \operatorname{Min}\left(P_{i}^{\max }, P_{i}^{o}+U R_{i}\right)
$$

\section{A. Classical PSO}

\section{Particle Swarm Optimization (PSO)}

A PSO is a population based modern heuristic search method that traces its evolution to the emergent motion of a flock of birds searching for food. It is a simple and powerful optimization tool which scatters random particles i.e. solutions into the problem space. These particles, called swarms collect information from each other through an array constructed by their respective positions. The particles update their positions by comparing their relative convergence towards the global optimum. The update mode is termed as the velocity of particles. Position and velocity are both updated in a heuristic manner using guidance from particles' own experience and the experience of its neighbors.

The position and velocity vectors of the $i$ th particle of a d-dimensional search space can be represented as $X_{i}=\left(x_{i 1}, x_{i 2}, \ldots \ldots \ldots \ldots x_{i d}\right)$ and $V_{i}=\left(v_{i 1}, v_{i 2}, \ldots \ldots . v_{i d}\right)$ respectively. On the basis of the value of the evaluation function, the best previous position of a particle is recorded and represented as pbest $t_{i}=\left(p_{i 1}, p_{i 2} \ldots \ldots . . p_{i d}\right)$. If the gth particle is the best among all particles in the group so far, it is represented as pbest $_{g}=$ gbest $=\left(p_{g 1}, p_{g 2}, \ldots \ldots \ldots . . p_{g d}\right)$. The particle tries to modify its position using the current velocity and the distance from pbest and gbest. The modified velocity and position of each particle for fitness evaluation in the next iteration are calculated using the following equations:

$$
\begin{aligned}
& v_{i d}^{k+1}=C\left[w^{*} v_{i d}^{k}+c_{1} * \operatorname{rand}_{1} *\left(\text { pbest }_{i d}-x_{i d}\right)+c_{2} * \operatorname{rand}_{2} *\left(\text { gbest }_{g d}-x_{i d}\right)\right] \\
& x_{i d}^{k+1}=x_{i d}+v_{i d}^{k+1}
\end{aligned}
$$

Here $\mathrm{C}$ is constriction factor, $w$ is the inertia weight parameter, $c_{1}, c_{2}$ are cognitive and social coefficients, and rand $_{1}$, rand $_{2}$ are random numbers between 0 and 1 . The inertia weight $w$ regulates the tradeoff between the global and local exploration capabilities of the particle. A large inertia weight helps in good global search, while a smaller value facilitates local exploration. Therefore, the practice is to use larger inertia weight factor during initial exploration and gradual reduction of its value as the search proceeds in further iterations. 


\section{B. New PSO with Time-Varying Acceleration Coefficients (NPSOTVAC)}

In PSO, tuning of parameters with time plays an important role in finding the optimum solution accurately and efficiently [4-6]. A new PSO technique where acceleration coefficients are varied with time are used in this paper to solve the complex problem of ED with valve point loading effects.

Kennedy and Eberhart [9] stated that a relatively higher value of the cognitive component, compared with the social component, results in roaming of individuals through a wide search space. On the other hand, a relatively high value of the social component leads particles to a local optimum prematurely.

In population-based optimization methods, the policy should be to encourage the individuals to roam through the entire search space, during the initial part of the search, without clustering around local optima. During the latter stages, however convergence towards the global optima should be encouraged, to find the optimum solution efficiently.

The idea behind TVAC is to enhance the global search in the early part of the optimization and to encourage the particles to converge towards the global optima at the end of the search. This is achieved by changing the acceleration coefficients $c_{1}$ and $c_{2}$ with time in such a manner that the cognitive component is reduced while the social component is increased as the search proceeds.

The acceleration coefficients are expressed as:

$$
\begin{aligned}
& c_{1}=\left(c_{1 F}-c_{1 I}\right) \frac{\text { iter }}{\text { iter }_{\max }}+c_{1 I} \\
& c_{2}=\left(c_{2 F}-c_{2 I}\right) \frac{\text { iter }_{\text {iter }_{\max }}}{\text { ite }_{2 I}}
\end{aligned}
$$

Where $c_{I I}, c_{I F}, c_{2 I}$ and $c_{2 F}$ are initial and final values of cognitive and social acceleration factors respectively. The flowchart of proposed NPSOTVAC algorithm is depicted in Fig 3.

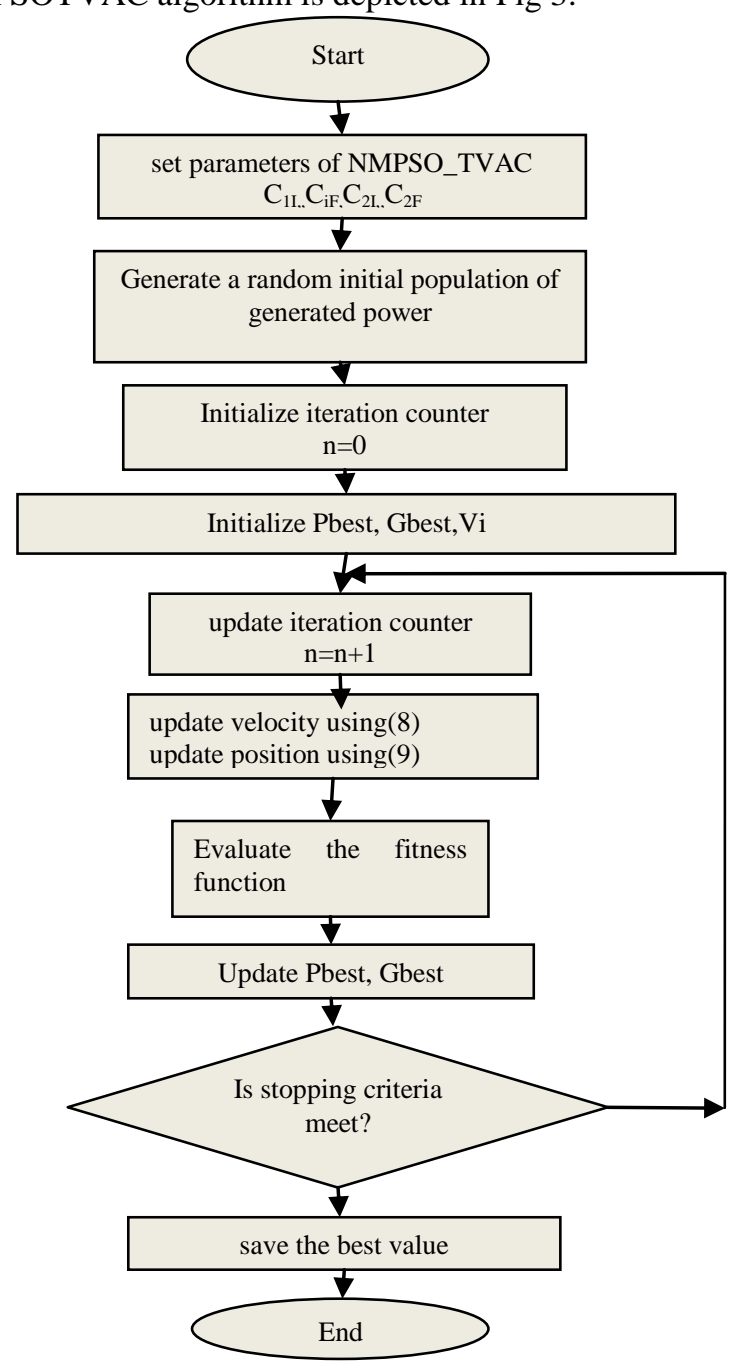

Figure 3. Flowchart of NPSOTVAC 


\section{Numerical Results and Analysis}

In order to show how to use the NPSOTVAC to solve NCED problems and to verify the effectiveness, two practical systems were employed to test the algorithm. The ramp rate limit, prohibited operating zones and valve point loading effects are considered for practical application.

\section{A. The two power systems}

Test system 1: The system consists of 6 generating units, 26 buses and 46 transmission lines [19-20].The load demand is 1263 MW.The generating capacity and cost coefficients, loss coefficients B, ramp rate limit and prohibited zones for six unit system are given in table I, II and III respectively.

Test system 2: This is a system with 15 generating units whose characteristics are given in Tables IV and V [21]. The load demand of the system is $2630 \mathrm{MW}$.

TABLE I. GENERATING UNIT CAPACITY AND COEFFICIENTS OF THE 6-UNIT SYSTEM

\begin{tabular}{|c|c|c|c|c|c|}
\hline Unit & $P_{i}^{\min }$ & $P_{i}^{\max }$ & $a_{i}$ & $b_{i}$ & $c_{i}$ \\
\hline 1 & 100 & 500 & 240 & 7.0 & 0.0070 \\
\hline 2 & 50 & 200 & 200 & 10.0 & 0.0095 \\
\hline 3 & 80 & 300 & 220 & 8.5 & 0.0090 \\
\hline 4 & 50 & 150 & 200 & 11.0 & 0.0090 \\
\hline 5 & 50 & 220 & 220 & 10.5 & 0.0080 \\
\hline 6 & 50 & 120 & 190 & 12.0 & 0.0075 \\
\hline
\end{tabular}

TABLE II. LOSS COEFFICIENTS B OF THE 6-UNIT SYSTEM

\begin{tabular}{|c|c|c|c|c|c|c|}
\hline$B_{i j}$ & 1 & 2 & 3 & 4 & 5 & 6 \\
\hline 1 & 0.0017 & 0.0012 & 0.0007 & -0.0001 & -0.0005 & -0.0002 \\
\hline 2 & 0.0012 & 0.0014 & 0.0009 & 0.0001 & -0.0006 & -0.0001 \\
\hline 3 & 0.0007 & 0.0009 & 0.0031 & 0 & -0.001 & -0.0006 \\
\hline 4 & -0.0001 & 0.0001 & 0 & 0.0024 & -0.0006 & -0.0008 \\
\hline 5 & -0.0005 & -0.0006 & -0.001 & -0.0006 & 0.0129 & -0.0002 \\
\hline 6 & -0.0002 & -0.0001 & -0.0006 & -0.0008 & -0.0002 & 0.0150 \\
\hline$B_{0 i}$ & -0.0004 & -0.0001 & 0.0007 & 0.0001 & 0.0002 & -0.0007 \\
\hline$B_{00}$ & \multicolumn{6}{|c|}{0.056} \\
\hline
\end{tabular}

TABLE III. GENERATING UNIT RAMP RATE LIMITS AND PROHIBITED ZONES OF THE 6-UNIT SYSTEM

\begin{tabular}{|c|l|c|c|c|}
\hline Unit & $P_{i}^{0}$ & $U R_{i}$ & $D R_{i}$ & Prohibited Zones \\
\hline 1 & 440 & 80 & 120 & {$[210,240][350,380]$} \\
\hline 2 & 170 & $\mathbf{5 0}$ & 90 & {$[90,110][140,160]$} \\
\hline 3 & 200 & 65 & 100 & {$[150,170][210,240]$} \\
\hline 4 & 150 & 50 & 90 & {$[80,90][110,120]$} \\
\hline 5 & 190 & 50 & 90 & {$[90,110][140,150]$} \\
\hline 6 & 110 & 50 & 90 & {$[75,85][100,105]$} \\
\hline
\end{tabular}

TABLE IV.GENERATING UNIT CAPACITY AND COEFFICIENTS OF THE 15-UNIT SYSTEM

\begin{tabular}{|c|c|c|c|c|c|}
\hline Unit & $P_{i}^{\min }$ & $P_{i}^{\max }$ & $a_{i}$ & $b_{i}$ & $c_{i}$ \\
\hline 1 & 150 & 455 & 671 & 10.1 & 0.000299 \\
\hline 2 & 150 & 455 & 574 & 10.2 & 0.000183 \\
\hline 3 & 20 & 130 & 374 & 8.8 & 0.001126 \\
\hline 4 & 20 & 130 & 374 & 8.8 & 0.001126 \\
\hline 5 & 150 & 470 & 461 & 10.4 & 0.000205 \\
\hline 6 & 135 & 460 & 630 & 10.1 & 0.000301 \\
\hline 7 & 135 & 465 & 548 & 9.8 & 0.000364 \\
\hline 8 & 60 & 300 & 227 & 11.2 & 0.000338 \\
\hline 9 & 25 & 162 & 173 & 11.2 & 0.000807 \\
\hline 10 & 25 & 160 & 175 & 10.7 & 0.001203 \\
\hline 11 & 20 & 80 & 186 & 10.2 & 0.003586 \\
\hline 12 & 20 & 80 & 230 & 9.9 & 0.005513 \\
\hline 13 & 25 & 85 & 225 & 13.1 & 0.000371 \\
\hline 14 & 15 & 55 & 309 & 12.1 & 0.001929 \\
\hline 15 & 15 & 55 & 323 & 12.4 & 0.004447 \\
\hline
\end{tabular}


A New modified particle swarm Optimization(PSO) Technique for Non-Convex Economic Dispatch

TABLE V. GENERATING UNIT RAMP RATE LIMITS AND PROHIBITED ZONES OF THE 15-UNIT SYSTEM

\begin{tabular}{|c|c|c|c|c|}
\hline Unit & $P_{i}^{0}$ & $U R_{i}$ & $D R_{i}$ & Prohibited Zones \\
\hline 1 & 400 & 80 & 120 & \\
\hline 2 & 300 & 80 & 120 & {$[185,225][305,335][420,450]$} \\
\hline 3 & 105 & 130 & 130 & \\
\hline 4 & 100 & 130 & 130 & \\
\hline 5 & 90 & 80 & 120 & {$[180,200][305,335][390,420]$} \\
\hline 6 & 400 & 80 & 120 & {$[230,255][365,395][430,455]$} \\
\hline 7 & 350 & 80 & 120 & \\
\hline 8 & 95 & 65 & 100 & \\
\hline 9 & 105 & 60 & 100 & \\
\hline 10 & 110 & 60 & 100 & \\
\hline 11 & 60 & 80 & 80 & \\
\hline 12 & 40 & 80 & 80 & {$[30,40][55,65]$} \\
\hline 13 & 30 & 80 & 80 & \\
\hline 14 & 20 & 55 & 55 & \\
\hline 15 & 20 & 55 & 55 & \\
\hline
\end{tabular}

\section{B. Results and discussion:}

NCED problem was solved using the NPSOTVAC. Optimum population size for 6 and 15 unit systems have been found 50 and 100. The performance of each system has been judged out of 50 trials. The acceleration coefficients $c_{1}$ (cognitive component) is initially set with high value and decreases as the search advances to explore the problem space in the first half of the search. In the later half, social component $c_{2}$ increases to exploit the search space and reach global optimal solution.

The results for 6 and 15 unit system are given in Table VI and Table VII. The minimum cost of operation obtained for 6-unit system is $15447.028(\$ / \mathrm{h})$ and for 15 unit system is $32450.39(\$ / \mathrm{h})$.

TABLE VI. GENERATOR OUTPUT FOR MINIMUM OPERATING COST (6-GENERATING UNIT SYSTEM)

\begin{tabular}{|l|l|}
\hline Unit Power Output & $\begin{array}{l}\text { NPSOTVAC } \\
(\$ / \mathrm{h})\end{array}$ \\
\hline P1(MW) & 450.01 \\
\hline P2(MW) & 171.18 \\
\hline P3(MW) & 266.543 \\
\hline P4(MW) & 131.916 \\
\hline P5(MW) & 165.58 \\
\hline P6(MW) & 89.62 \\
\hline Total power output(MW) & 1274.849 \\
\hline Total loss(MW) & 12.44 \\
\hline Total generation cost(\$/h) & 15445.028 \\
\hline
\end{tabular}

TABLE VII. GENERATOR OUTPUT FOR MINIMUM OPERATING COST (15- GENERATING UNIT SYSTEM)

\begin{tabular}{|l|l|}
\hline Unit Power Output & $\begin{array}{l}\text { NPSOTVAC } \\
(\$ / \mathrm{h})\end{array}$ \\
\hline P1(MW) & 455 \\
\hline P2(MW) & 375 \\
\hline P3(MW) & 130 \\
\hline P4(MW) & 135.28 \\
\hline P5(MW) & 165.77 \\
\hline P6(MW) & 460 \\
\hline P7(MW) & 424.52 \\
\hline P8(MW) & 65 \\
\hline P9(MW) & 25 \\
\hline P10(MW) & 157 \\
\hline P11(MW) & 84.23 \\
\hline P12(MW) & 74.68 \\
\hline P13(MW) & 25 \\
\hline P14(MW) & 24.98 \\
\hline
\end{tabular}




\begin{tabular}{|l|l|}
\hline P15(MW) & 34 \\
\hline Total power output(MW) & 2635.46 \\
\hline Total generation $\operatorname{cost}(\$ / \mathrm{h})$ & 32450.39 \\
\hline
\end{tabular}

\section{Convergence characteristics}

The convergence characteristics of NPSOTVAC for 6 and 15 unit system is given in Fig 4 and Fig 5.The two figures show that the characteristics of NPSOTVAC, are continuously decreasing and tending to converge to find global optimal solution.

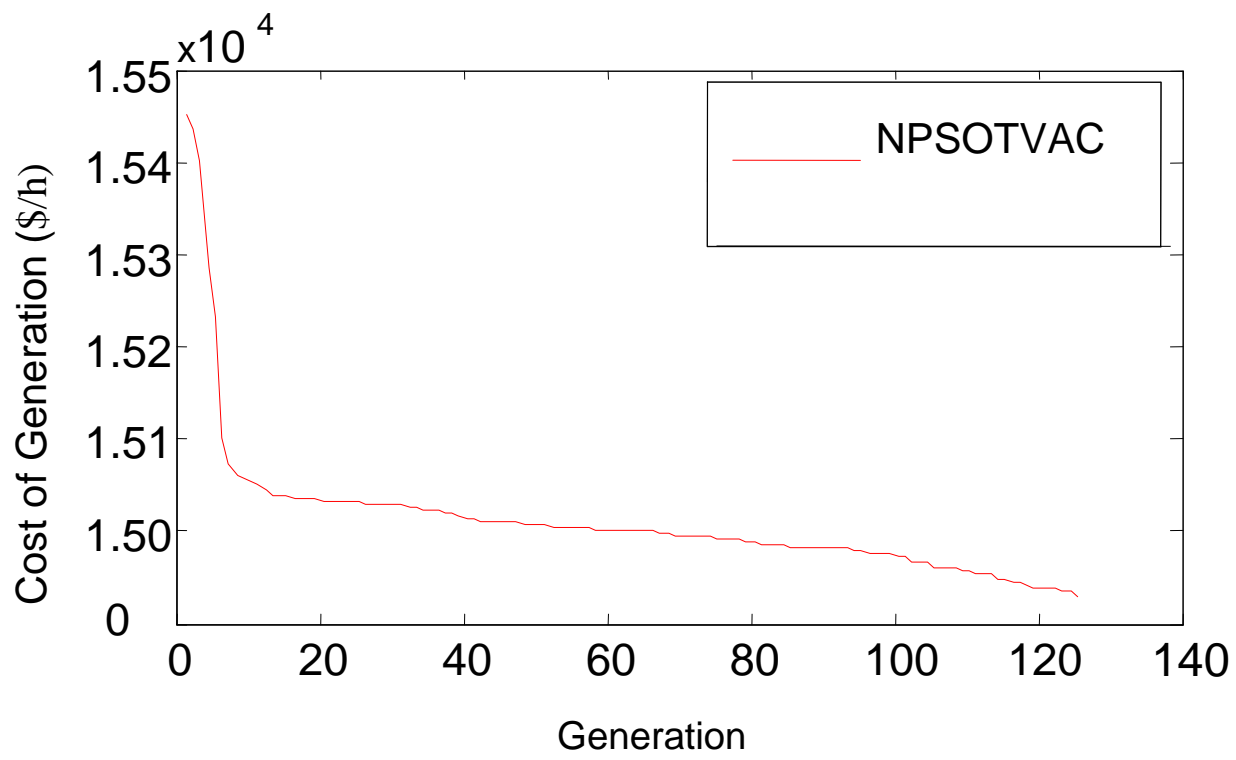

Fig 4.Convergence properties of 6-unit generating system using NPSOTVAC

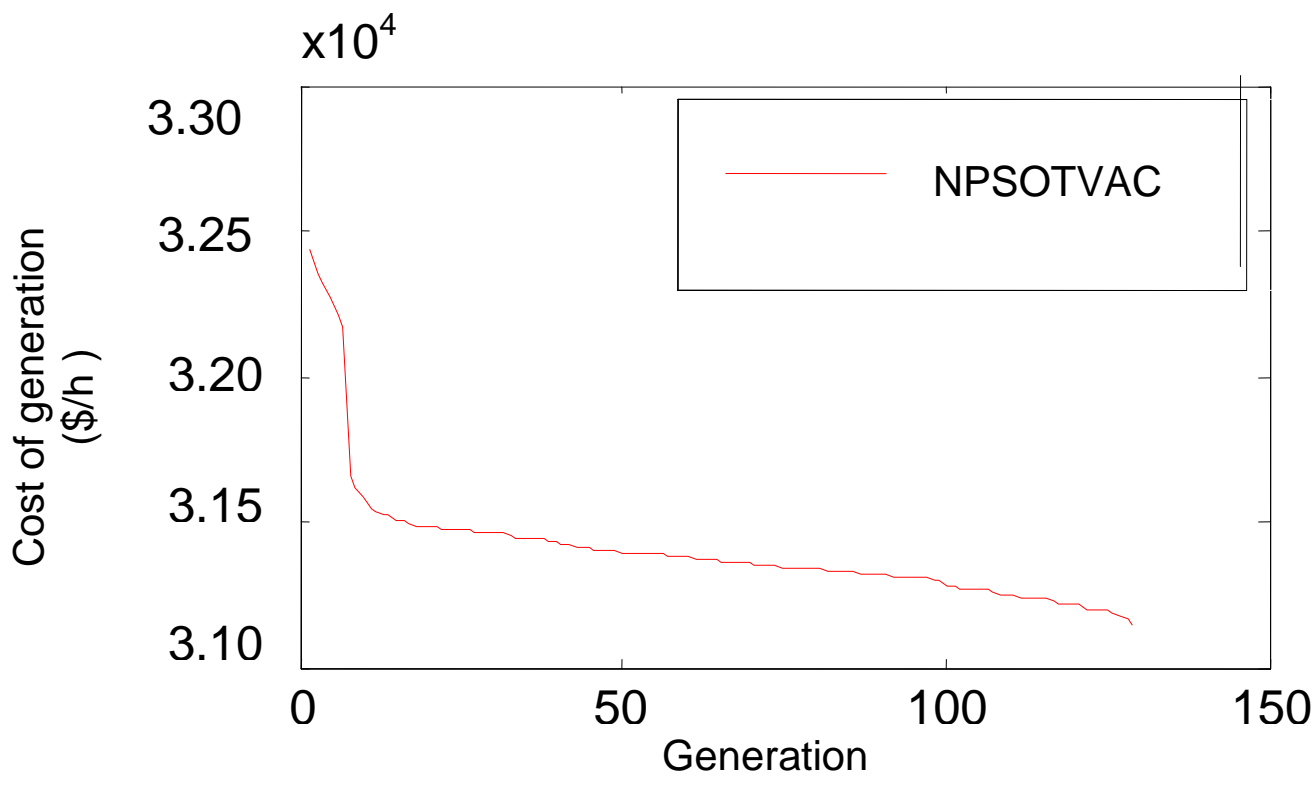

Fig 5.Convergence properties of 15-unit generating system using NPSOTVAC

\section{Conclusion}

The complex and nonconvex economic load dispatch is solved by using new modified PSO technique. By proper tuning of parameters a balance between local and global parameters are set which helps in addressing problem of premature convergence. The results reflect the efficiency of this method. It is simple, less time consuming and gives better solution. 


\section{REFERENCES}

[1]. A.J. Wood and B.F. Wollenberg, "Power generation, operation and control", (Book), New York, Wiley, 1984.

[2]. D.C. Walter and G. B. Sheble, "Gentic algorithm solution of economic load dispatch with valve point loading" ,IEEE Transactions on Power Systems., vol. 8, pp.1325-1332, Aug 1993.

[3]. S.O.Orero and M.R. Irving, "Economic dispatch of generators with prohibited operating zones: a genetic algorithm approach", IEE proceedings, Generation, transmission and Distribution, vol. 143, No. 6, November 1996.

[4]. Z.L. Gaing, "Particle swarm optimization to solving the economic dispatch considering generator constraints", IEEE Trans. Power system,vol. 18, no. 3, August 2003, pp.1718-1727.

[5]. J.B. Park, K.S. Lee, J.R. Shin and K.Y. Lee, "A Particle swarm optimization for Economic Dispatch with non-smooth cost functions", IEEE Trans. Power system,.vol. 20, no. 1, February 2005, pp.34-42.

[6]. A. ImmanualSelvakumar and K. Thanushkodi, "A new Particle swarm optimization solution to nonconvex economic dispatch problems”, IEEE Trans. On Power system,.vol. 22, no. 1, February 2007, pp.42-51.

[7]. T. Aruldoss, A. Victoire and A. E. Jeyakumar, "Reserve constrained dynamic dispatch of units with valve point effects", IEEE Transactions on Power Systems, vol. 20, no. 3, August 2005, pp. 1273-1282.

[8]. Safari A, Shayeghi H," Iteration particle swarm optimization procedure for economic load dispatch with generator constraints", Expert Systems Appl. vol. 38, pp. 6043-6048, 2011

[9]. J.Kennedy and R. Eberhart, "Particle swarm optimization", in proc. IEEE Conf. on Neural Networks (ICNN'95), vol. IV, Perth, Australia, 1995, pp.1942-1948.

[10]. Y. Shi and R.C. Eberhart, "Empirical study of particle swarm optimization", Proc. of IEEE International congress on Evolutionary Computation, , vol. 3, 1999, pp. 101-106.

[11]. R.C. Eberhart and Y. Shi, "Comparing inertia weights and constriction factors in particle swarm optimization", Proceedings of the Congress on Evolutionary Computation,, vol. 1, 2000, pp. 84-88.

[12]. Y. Shi and R.C. Eberhart, "Fuzzy adaptive particle swarm optimization", in Proc. IEEE International Conference on Evolutionary Computation, 2001, pp.101-106.

[13]. John G. Vlachogiannis and Kwang Y. Lee, "A comparative study on particle swarm optimization for optimal steady state performance of power systems", IEEE Transactions on Power Systems, vol. 21, no. 4, Nov. 2006, pp. 1718-1727.

[14]. C. Kuo, "A novel coding scheme for practical economic dispatch by modified particle swarm approach," IEEE Trans. Power Syst., vol. 23 , no. 4, pp. 1825-1835, Nov. 2008.

[15]. Krishna TeerthChaturvedi, M. Pandit, and L. Srivastava, "Self-organizing hierarchicalparticle swarm optimization for nonconvex economic dispatch,"IEEE Trans. Power Syst., vol. 23, no. 3, pp. 1079-1087, Aug. 2008

[16]. B. K. Panigrahi, V. R. Pandi, and S. Das, "Adaptive particle swarm optimization approach for static and dynamic economic load dispatch," Energy Convers. Manage., vol. 49, no. 6, pp. 1407-1415, 2008.

[17]. J. G. Vlachogiannis and K. Y. Lee. Economic Load Dispatch-A Comparative Study on Heuristic Optimization Techniques With an Improved Coordinated Aggregation-Based PSO . IEEE Trans. Power Syst., vol. 24, no. 2, pp. 991-1001, May.2009.

[18]. Krishna TeerthChaturvedi, ManjareePandit and LaxmiSrivastava, "Particle Swarm Optimization with Time Varying Acceleration Coefficients for Nonconvex Economic Power Dispatch"International Journal of Electrical Power and Energy systems, vol. 31, pp. 249-257, 2009.

[19]. P.-H. Chen and H.-C. Chang, "Large-scale economic dispatch by genetic algorithm”, IEEE Trans. Power Syst. ,vol. 10, no.4, pp.1919-1926, Nov. 1995.

[20]. H. Yoshida, K. Kawata, Y. Fukuyama, S. Takayama, and Y. Nakanishi, "A particle swarm optimization for reactive power and voltage control considering voltage security assessment," IEEE Trans. Power Syst., vol. 15, pp. 1232-1239, Nov. 2000.

[21]. F. N. Lee and A. M. Breipohl, "Reserve constrained economic dispatch with prohibited zones," IEEE Trans. Power Syst., vol. 8, pp. 246-254, Feb. 1993 